\title{
HEURISTIC IMAGE RECOGNITION
}

\author{
Cosol, A. C.; Vlad, M. S. \& SgarciU, V.
}

Abstract: Using a distance specifically designed for AntiSpam filters, we developed an image retrieval system that can extract similar images ignoring most noise types. Sometimes, when taking a picture, random pixels change, or different objects appear or disappear from the image. When retrieval is performed, these minor modifications can cause serious problems. Using SID (short for spam image distance) our approach is insensitive to noise and combined to other image characteristics can boost up performance to any image retrieval algorithm.

Key words: SID, histogram, image retrieval, antispam
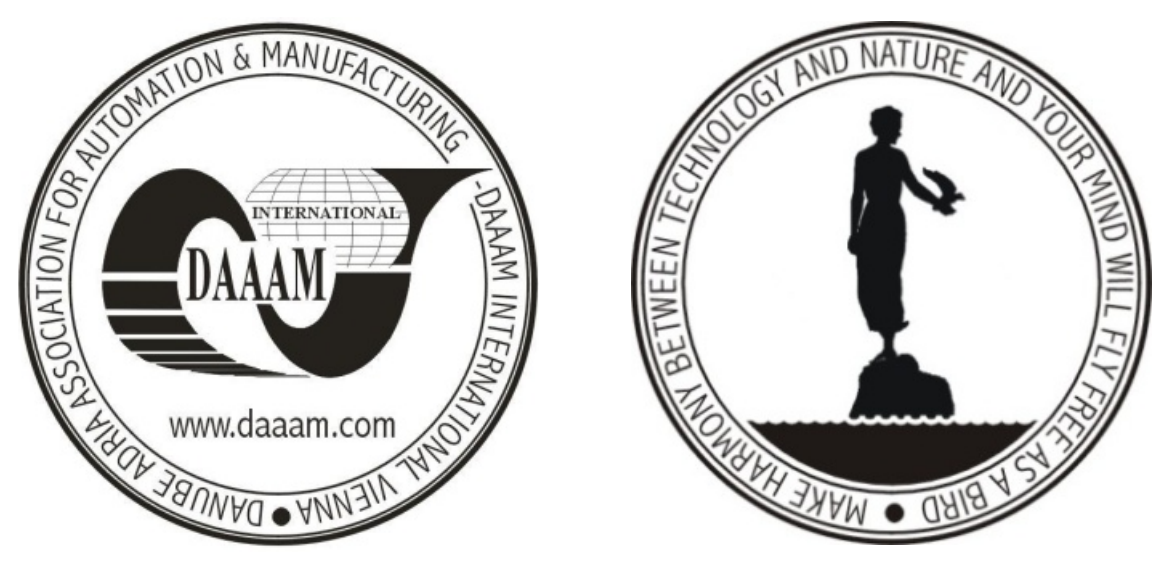

Authors' data: PhD Eng. Cosoi, A[lexandru] C[atalin]; Dr. Eng. Vlad, M[adalin] S[tefan]; Prof. Dr. Eng. Sgarciu, V[alentin], University „Politehnica“ Bucharest, Romania, Splaiul Independentei 313, Bucharest, PO BOX 060042; catalin.cosoi@gmail.com, madalinv@ac.pub.ro,vsgarciu@cs.pub.ro

This Publication has to be referred as: Cosoi, A[lexandru] C[atalin]; Vlad, M[adalin] S[tefan] \& Sgarciu V[alentin] (2009). Heuristic Image Recognition, Chapter 39 in DAAAM International Scientific Book 2009, pp. 377-384, B. Katalinic (Ed.), Published by DAAAM International, ISBN 978-3-901509-69-8, ISSN 17269687, Vienna, Austria

DOI: $10.2507 /$ daaam.scibook.2009.39 